Urologe 2012 · 51:310-318

DOI 10.1007/s00120-012-2833-6

Online publiziert: 10. März 2012

๑) Springer-Verlag 2012

\begin{abstract}
Das Urothelkarzinom der Harnblase stellt mit einer Inzidenz von 2530 Neuerkrankungen/100.000 Einwohner/Jahr für den Urologen die häufigste maligne Erkrankung nach dem Prostatakarzinom dar. Weiterhin gilt die radikale Zystektomie mit bilateraler pelviner Lymphadenektomie (LAD) als Goldstandard der Therapie bei den muskelinvasiven Karzinomen. Studien zeigen, dass zwischen 14-30\% der Patienten mit muskelinvasivem Urothelkarzinom der Harnblase zum Zeitpunkt der Zystektomie bereits unerwartet Lymphknoten(LK-)Metastasen aufweisen, wobei die Inzidenz mit dem Tumorstadium proportional ansteigt. Finden sich bei ca. $18 \%$ der Patienten mit einem pT2Stadium LK-Metastasen, so steigt diese Zahl bei pT3a- auf $26 \%$, bei pT3bauf $46 \%$ und bei pT4-Stadien auf $42 \%$ an $[10,17,29]$.
\end{abstract}

Bereits 1951 stellten Kerr u. Colby [15] fest, dass die Lokalrezidivrate bei Patienten mit kombinierter Zystektomie und LAD niedriger ausfiel als bei denen mit alleiniger Zystektomie. 1982 beschrieb Skinner [27] einen Überlebensvorteil für die Patienten, die sich einer sorgfältigen LAD unterzogen. Bis heute wird davon ausgegangen, dass u. a. in situ belassene tumorpositive $\mathrm{LK}$ als Ausgangspunkt für einen Tumorprogress bzw. -rezidiv verantwortlich sind.

T. Metzger · G.N. Thalmann · P. Zehnder

Urologische Universitätsklinik, Inselspital, Bern

\title{
Lymphadenektomie im Rahmen der radikalen Zystektomie
}

\section{Aktueller Stand und Kontroversen}

Es liegen zahlreiche retrospektive Studien vor, die den Benefit einer LAD hinsichtlich dem Gesamtüberleben zeigen. Daneben wird durch die LAD ein genaueres postoperatives Staging sowie eine prognostische Aussage möglich, mit Risikostratifizierung hinsichtlich Tumorrezidiv und entsprechender Selektion von $\mathrm{Pa}$ tienten für eine adjuvante Therapie. Die LAD ist somit zwingend als Standardverfahren im Rahmen der Zystektomie durchzuführen. Daneben gibt es aber etliche Kontroversen, wovon wir einige in diesem Artikel aufgreifen wollen.

\section{Lymphabfluss der Harnblase und LAD-Templates}

Rein historisch wird als „Standardtemplate" die Resektion des lymphatischen Gewebes zwischen Blase und Beckenwand, entlang der iliakal externen und internen Gefäße bis hin zum oberen Drittel der iliakal kommunen Gefäße inklusive Fossa Marcille gesehen [33]. Da aber vielerorts nur gerade eine limitierte $\mathrm{LAD}$ durchgeführt wird, ist in der neueren Literatur eine limitierte LAD gemeint, wenn von einem "Standardtemplate“ gesprochen wird. Prinzipiell werden drei Templates unterschieden und in den verschiedenen Kliniken entsprechend angewandt: 1. „limited“ (eingeschränkt), 2. „extended“ (ausgedehnt) und 3. „super-extended“ (maximal ausgedehnt) Template (• Abb. 1).
Wichtige Informationen bezüglich des lymphogenen Metastasierungsmusters erbrachten die sog. Mapping-Studien [1, 24, 31]. Im Rahmen dieser Analysen wurde das lymphatische Gewebe nach Lokalisationen getrennt dem Pathologen eingesandt und aufgearbeitet. Obwohl diese Untersuchungen aufgrund der operateurabhängigen Lokalisationsbezeichnung ein beträchtliches Fehlerpotential aufweisen, können folgende Aussagen gemacht werden:

- Auch bei strikt unilateralen Tumoren ist eine bilaterale/kontralaterale Metastasierung häufig.

- Das Auftreten von solitären extrapelvinen Metastasen („skipped lesions“) ist - wenn es überhaupt vorkommt äußerst selten.

Um eine untersucherunabhängige Aussage bzgl. primärem Lymphabfluss bzw. primären Lymphknotenstationen der Harnblase machen zu können, führten Roth et al. [24] eine technetiumbasierte Mapping-Studie durch. Dabei wurde ein Tag präoperativ Technetiumnanokolloid in die tumorfreie Seite der Harnblase injiziert. Gleichentags erfolgte eine Detektion der radioaktiv markierten LK mittels SPECT („single photon emission computed tomography“) mit anschließender Fusionsbildgebung mit konventionellem CT. Bei den 60 so untersuchten Patienten wurden im Rahmen der Zystektomie die technetiumpositiven LK mittels Gamma- 
Hier steht eine Anzeige.

黛 Springer 


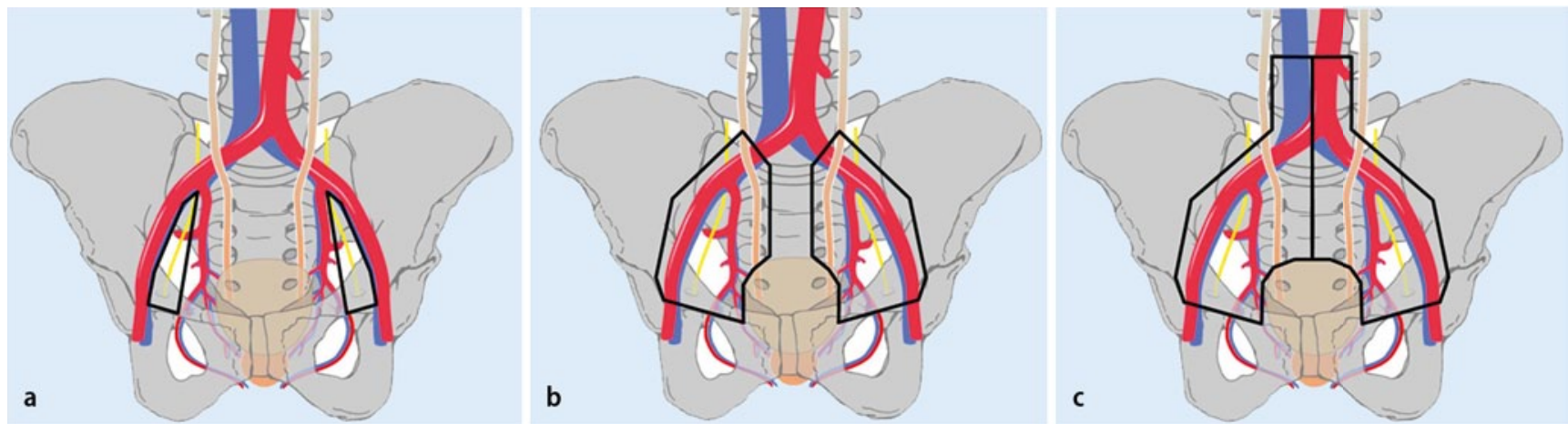

Abb. $1 \Delta$ Die verschiedenen Templates: a eingeschränkt (, limited“), b ausgedehnt („extended“), c maximal-ausgedehnt (,super extended“). (Mod. nach [36], mit freundl. Genehmigung von Elsevier)

sonde aufgesucht und separat zur histologischen Aufarbeitung eingeschickt, gefolgt von einer ausgedehnten Back-upLAD. Im Median ließen sich pro Patient 4 (1-14) radioaktiv markierte, sog. primäre lymphatische Landezonen-LK auffinden, von denen wiederum nur $8 \%$ oberhalb der ureteroiliakalen Kreuzung angesiedelt waren. 30\% der LK fanden sich iliakal extern (davon $12 \%$ in der Fossa Marcille), 26\% iliakal intern, 25\% im Bereich der Fossa obturatoria, 15\% iliakal kommun und $4 \%$ paraaortal/paracaval. Keiner der Patienten wies solitäre extrapelvine technetiumpositive LK auf. Andere Publikationen stützen diese Beobachtung, so dass die Autoren die proximale Ausdehnung der LAD bis zur Kreuzung des Ureters mit den kommunen Iliakalgefäßen als sinnvoll und ausreichend erachten. Dies insbesondere hinsichtlich der mit einer darüber hinausgehenden LAD erhöhten Gefahr iatrogener Kollateralschäden, in erster Linie durch eine Verletzung der autonomen Nerven. Dagegen lohnt sich die feinsäuberliche Resektion der medial der iliakal internen Gefäße gelegenen LK. Hier finden sich $11 \%$ der primären lymphatischen Landezonen.

Dies wird unterstrichen durch die Untersuchung von Seiler et al. [26]. Sie analysierten die LK-Präparate von 162 zystektomierten Patienten hinsichtlich ihrer Verteilung und stellten fest, dass die im Median 25 (8-55) je Patient resezierten LK zu 39\% in der Region iliakal extern, $35 \%$ obturatorisch und $26 \%$ iliakal intern aufzufinden waren. Hinsichtlich der Verteilung der 625 LK-Metastasen von insgesamt 4080 untersuchten LK zeigte sich kein signifikanter Unterschied (33\% ilia- kal extern, 38\% obturatorisch, 29\% iliakal intern). Dagegen fand sich in $10 \%$ der Patienten mit einer unifokalen LK-Metastase der Befund im Bereich iliakal intern.

Das Belassen der iliakal internen LK respektive die Anwendung einer limitierten LAD ist aus onkologischer Sicht insuffizient. Dhar et al. [5] verglichen die ausgedehnte LAD, wie sie z. B. an der Berner Universitätsklinik durchgeführt wird, mit der limitierten Variante, die an der „Cleveland Clinic" praktiziert wurde. Dies war ein retrospektiver Vergleich beider Zentren mit 322 Patienten im Berner und 336 im Cleveland-Kollektiv. Anhand dieser Daten lässt sich der diagnostische Vorteil einer ausgedehnten LAD dokumentieren, da sich hierdurch im Vergleich zur eingeschränkten LAD eine Verdoppellung der $\mathrm{pN}+$-Stadien (13\% vs. $26 \%$ ) ergibt. Konsekutiv liegt dann auch das rezidivfreie 5-Jahres-Überleben der pN +-Patienten bei der limitierten LAD bei 7\%, bei der ausgedehnten LAD bei $35 \%$, bei Patienten mit einem pT2pN0-2-Tumor bei 63\% vs. $71 \%(\mathrm{p}=0,22)$ und bei Patienten mit einem pT3pN0-2-Tumor bei $19 \%$ vs. $49 \%$ $(\mathrm{p}<0,0001)$ jeweils zugunsten der ausgedehnten LAD.

Die ähnliche Nachfolgestudie hilft, die proximale Begrenzung des ausgedehnten LAD-Templates festzulegen. In dieser großen Vergleichsstudie zweier Zystektomiezentren [University of Southern California (USC) vs. Bern] wurden retrospektiv die Daten von 554 (USC) und 405 (Bern) Zystektomiepatienten bezüglich Ausdehnung der LAD und onkologischem Outcome analysiert [36]. Während an der USC die maximal ausgedehnte LAD bis zur A. mesenterica in- ferior hin propagiert wird, erfolgt diese in Bern bis zur Kreuzung des Ureters mit den iliakal kommunen Gefäßen. Erwartungsgemäß fanden sich an der USC mehr resezierte LK (Median: 38 vs. $22 \mathrm{LK}$; $\mathrm{p}<0,0001)$. Auch lag die Inzidenz detektierter LK-Metastasen höher (35\% vs. $28 \% ; \mathrm{p}<0,02)$. Hingegen konnte kein signifikanter Unterschied bzgl. rezidivfreiem und Gesamtüberleben gefunden werden. Die Autoren folgerten hieraus, dass die LAD im ausgedehnten Template bis zum oberen Drittel der iliakal kommunen Gefäße ein vergleichbares Outcome generiert wie das maximal ausgedehnte Template. Entscheidender als die alleinige Anzahl resezierter $\mathrm{LK}$ ist die sorgfältige $\mathrm{LAD}$ im ausgedehnten Template. Die Resektion CT-graphisch oder makroskopisch intraoperativ suspekter LK außerhalb des ausgedehnten Templates respektive eine Ausdehnung nach kranial z. B. bei Patienten mit Status nach neoadjuvanter Chemotherapie wegen nachgewiesenen pelvinen LK-Metastasen, kann in einzelnen Fällen trotzdem sinnvoll sein.

\section{Lymphadenektomie und Morbidität}

Eine sorgfältige und ausgedehnte LAD ist ein zeitintensiver Teilschritt bei der radikalen Zystektomie. Es werden deshalb immer wieder Versuche unternommen, die LAD aus zeitlichen sowie morbiditätsbasierten Überlegungen abzukürzen. Dies war auch das Ziel der Multicenterstudie von Baltaci et al. [2], welche intraoperative Schnellschnittuntersuchungen durchführten und analysierten. Dabei wurden aber nur gerade die obtura- 
torischen LK untersucht. Bei gesamthaft 118 Patienten zeigte sich eine Sensitivität von $95 \% / 87 \%$ (rechts-/linksseitig), Spezifität $100 \% / 100 \%$, positiver prädiktiver Wert $100 \% / 100 \%$ und negativer prädiktiver Wert 99\%/98\%. Bei 15 Patienten (18\%) mit negativen obturatorischen LK fanden sich LK-Metastasen an anderen Lokalisationen. Somit muss bei Verwendung dieser Methode mit einem inakzeptablen Understaging gerechnet werden. Auch ist aufgrund der von Roth et al. [24] demonstrierten Komplexität des Lymphabflusses eine alleinige Schnellschnittuntersuchung der obturatorischen LK wohl als unzureichend zu betrachten.

Eine weitere Methode zur Evaluation, inwiefern eine ausgedehnte LAD beim jeweiligen Patienten überhaupt notwendig ist, ist die Untersuchung des Sentinel-LK. Dieses Verfahren ist bei anderen Tumorentitäten wie dem malignen Melanom oder dem Mammakarzinom etabliert. Liedberg et al. [18] verfolgten ein gleiches Konzept beim muskelinvasiven Harnblasentumor und injizierten zystoskopisch 75 Patienten $70 \mathrm{MBq}\left({ }^{99 \mathrm{~m}}\right)$ Technetiumnanokolloid peritumoral. Es erfolgte dann eine präoperative sowie intraoperative Lymphoszintigraphie und Methylenblaudetektion. Auf diese Weise konnten sie bei $87 \%$ des Patientenkollektivs einen Sentinel-LK detektieren. Jedoch wiesen nur 26 der 32 LK-positiven Patienten (81\%) einen ebensolchen positiven Sentinel-LK auf, woraus eine falschnegative Rate von beträchtlichen $19 \%$ resultiert.

Die bereits erwähnte ebenfalls technetiumbasierte Mapping-Studie von Roth et al. [24] widerspricht auch der Idee eines Sentinel-LK-Verfahrens. Im Rahmen einer Follow-up-Studie von 40 Patienten mit strikt unilateralem Harnblasentumor wiesen alle diese Patienten mindestens einen radioaktiv markierten LK auf der ipsilateralen Seite auf und es fand sich bei $40 \%$ zumindest ein technetiumpositiver LK auf der kontralateralen Seite.

Zweifelsohne geht eine ausgedehnte Lymphadenektomie mit einer signifikant erhöhten Operationsdauer einher und birgt Risiken und Komplikationen (Lymphozele, Verletzung von Nachbarstrukturen insbesondere der Gefäße und Nerven etc.). Andererseits sind diese Risiken auf-

Urologe 2012·51:310-318 DOI 10.1007/s00120-012-2833-6

C) Springer-Verlag 2012

\section{T. Metzger · G.N. Thalmann P P. Zehnder \\ Lymphadenektomie im Rahmen der radikalen Zystektomie. Aktueller Stand und Kontroversen}

\section{Zusammenfassung}

Die pelvine Lymphadenektomie (LAD) ist ein entscheidender Bestandteil der radikalen Zystektomie bei muskelinvasiven Harnblasentumoren. Die optimale Ausdehnung der LAD, insbesondere die proximale Ausdehnung, wird weiterhin kontrovers diskutiert. Aufgrund der vorliegenden Mappingsowie retrospektiven Studien scheint die ausgedehnte LAD bis zum oberen Drittel der iliakal kommunen Gefäße einen prognostischen wie auch therapeutischen Benefit mit sich zu bringen und sollte deshalb als Standard definiert werden. Dies gilt gleichermaßen für die offene wie auch für die minimal-invasive Chirurgie. Dabei ist die alleinige Lymphknoten- (LK-)Anzahl kein hinlängliches Qualitätskriterium für eine LAD, da die LK-Zahl zu sehr durch anatomische Variabilität, chirurgische Technik, Wahl des Templates, his- topathologische Aufarbeitung etc. beeinflusst wird und daraus konsekutiv beträchtliche interinstitutionelle Unterschiede resultieren, welche keinen Vergleich zulassen. Die LKDensity wird als besserer prognostischer Faktor postuliert, wird aber in gleichem Ausmaß von denselben Faktoren beeinflusst. Hinsichtlich molekularer Diagnostik zur Verbesserung der Sensitivität des LK-Stagings ist weitere Forschungstätigkeit notwendig. Es wird sich zeigen, inwieweit die Ergebnisse der beiden groß angelegten prospektiv randomisierten Studien zur Klärung der optimalen Ausdehnung der LAD beitragen werden.

\section{Schlüsselwörter}

Invasiver Harnblasentumor - Zystektomie . Pelvine Lymphadenektomie · Template . Outcome

\section{Lymphadenectomy for bladder cancer. Current status and controversies}

\section{Abstract}

Pelvic lymph node dissection is an integral part of the radical cystectomy procedure for patients with muscle-invasive bladder cancer. The optimal extent of the lymphadenectomy (LND) and mainly the proximal template boundary remain controversial issues. In view of the existing mapping studies and retrospective analyses, extended LND up to the mid-upper third of the common iliac vessels appears to provide further prognostic and therapeutic benefit and therefore should be defined as standard LND. This applies for all procedures irrespective of the choice of surgical approach (open surgery, minimally invasive approach). In this context total lymph node count is not a quality criterion because nodal yield is overly influenced by the indi- vidual patient's anatomy, surgical technique, template applied and pathological workup. Consecutively, considerable inter-institutional differences result, which render any comparison impossible. Lymph node density is thought to be a superior prognostic factor, but it is similarly influenced by the abovementioned factors. Concerning molecular techniques to improve the sensitivity of postoperative nodal staging further research is necessary. The two ongoing prospective randomized trials will potentially help to further define the optimal LND template.

\section{Keywords}

Invasive bladder cancer - Cystectomy · Pelvic lymph node dissection · Template · Outcome grund des zuvor erwähnten Benefits hinsichtlich des Überlebens klar zu rechtfertigen [4].

Leissner et al. [17] konnten zeigen, dass das Risiko einer Lymphozele respektive eines Lymphödems nach LAD mit $<16$ resezierten $L K$ vergleichbar mit dem von Patienten mit $\geq 16$ resezierten LK ist ( $2 \%$ vs.1,1\%). Auch Poulsen et al. [22] zeigten, dass die limitierte im Vergleich zur exten- dierten LAD zu keiner signifikanten Reduktion der Lymphozelenrate führt (1,5\% vs. 1,6\%). Generell findet sich kein signifikanter Unterschied bezüglich perioperativer Mortalität, frühen postoperativen Komplikationen und Bedarf an Bluttransfusionen [4]. 


\section{Lymphknotenanzahl - ein Qualitätsmerkmal?}

Eine bestimmte Anzahl an LK festzulegen, die im Rahmen der LAD reseziert werden sollten, um so einen Standard zu setzen und eine Prozessqualität zu generieren, ist ein überdenkenswerter Ansatz, dessen Grenzen aber rasch erkennbar sind. Die Zahl der resezierten LK hängt zunächst davon ab, wie viele LK der einzelne Patient überhaupt aufweist. Diesbezüglich wissen wir von einer relativ hohen interindividuellen Variabilität [32]. Auch spielt die Erfahrung des Operateurs sowie das verwendete Template eine entscheidende Rolle. Schließlich gilt es auch einen LK als solchen zu definieren und bereits hierbei finden sich unterschiedliche Definitionen. Während die einen Pathologen das Vorhandensein eines afferenten sowie efferenten Lymphgefäßes als zwingende Voraussetzung zur Definition eines LK postulieren, geben sich andere damit zufrieden, wenn sich von einer Kapsel umgebene Lymphozyten oder gar nur ein Lymphozytencluster abgrenzen lassen. Diese Abhängigkeit von Institutionsstandards konnte vor kurzem deutlich aufgezeigt werden. Trotz der Anwendung des exakt gleichen LAD-Templates resultierten signifikant verschiedene Anzahlen an resezierten LK [University of Southern California: 72 (29-179) LK; Oregon Health Sciences University: 40 (5-118) LK; $\mathrm{p}<0,001 ;$ [6]].

May et al. [19] arbeiteten in einer retrospektiven Studie die Daten von 1291 zystektomierten Patienten mit muskelinvasivem Urothelkarzinom der Harnblase auf, die allesamt einen pN0-Status aufwiesen. Anhand einer multivariaten Cox-Regressionsanalyse wurde die Anzahl LK ermittelt, die als unabhängiger Risikofaktor für das Tumor-spezifische Überleben stratifiziert. Hier zeigte sich ein tumorspezifisches 5 -Jahres-Überleben von $72 \%$ bei $<16$ resezierten $\mathrm{LK}$ zu $83 \%$ bei $\geq 16$ resezierten LK $(p=0,01)$. Andere Autoren konnten die Korrelation aus Anzahl LK und damit einhergehendem Überlebensvorteil nicht bestätigen $[8,21]$. Es dürfte sich um rein institutionelle Cut-offs handeln, welche nicht verglichen werden können.

Fleischmann et al. [8] untersuchten den Zusammenhang zwischen LK-An- zahl und rezidivfreiem sowie Gesamtüberleben anhand einer interquartilen Analyse. Hierzu wurde das Patientenkollektiv in Quartilen mit 10-17, 18-21, 2228 und 29-43 resezierten LK eingeteilt. Dabei zeigte sich weder bzgl. rezidivfreiem noch Gesamtüberleben ein signifikanter Unterschied zwischen den 4 Gruppen. Das vergleichbare Überleben unabhängig der Anzahl resezierter LK unterstreicht nochmals den Wert der gründlichen LAD im ausgedehnten Template.

In diesem Sinne ist die alleinige Anzahl der resezierten LK, die gerne als Vergleichs- oder Qualitätsparameter herangezogen wird, kein hinlängliches Qualitätskriterium der LAD, wie Fang et al. [7] zeigen konnten. Retrospektiv wurden dabei 349 Patienten aufgearbeitet, der Zeitraum erstreckte sich über 8 Jahre, wobei nach 4 Jahren etablierte wurde, dass die histologische Aufarbeitung mindestens 16 LK umfassen muss, ansonsten erfolgte eine nochmalige Untersuchung der LADPräparate. 147 Präparate wurden vor Einführung dieser Regelung und 202 danach untersucht, mit dem Ergebnis einer $\mathrm{Zu}$ nahme der verifizierten LK im Median von 15 auf 20 nach Einführung dieser Regelung. Zeigten sich vorher in $42,9 \%$ der Fälle $\geq 16 \mathrm{LK}$, so waren dies im Verlauf $69,3 \%(\mathrm{p}<0,01)$. Die Studie zeigt somit klar, dass die Zahl der LK nicht nur von der Gründlichkeit des Operateurs sondern auch vom Pathologen abhängig ist. Somit sind interinstitutionelle Vergleiche bzgl. Anzahl LK nur eingeschränkt aussagekräftig.

\section{Lymphknoten-Density und Lymphknoten mit extrakapsulärem Wachstum}

Die LK-Density ist definiert als Verhältnis aus Anzahl tumorpositiver LK und Anzahl gesamthaft resezierter LK. Die Idee, einen solchen Quotienten zu bilden, beruht auf den eben genannten Tatsachen, dass die alleinige LK-Zahl vom einzelnen Individuum, Operateur und Pathologen abhängig ist $[12,30]$ und somit durch die LK-Density ein unabhängiger Risikofaktor generiert werden kann. Stein et al. [28] konnten zeigen, dass Patienten mit einer LK-Density $\leq 20 \%$ ein rezidivfreies 10-Jahres-Überleben von $43 \%$ erreichten im Vergleich zu 17\% bei den Patienten mit einer LK-Density von $>20 \%$.

Auch in aktuelleren Studien [21, 34] wird die LK-Density als unabhängiger prädiktiver Faktor für das Überleben gesehen und deshalb die Integrierung in das pathologische Staging gefordert [13, 23]. Jedoch gilt es zu bedenken, dass auch die LK-Density der Ausdehnung der LAD Rechnung trägt und der Nenner der Gleichung aufgrund des vorgängig geschriebenen deutlichen interinstitutionellen Schwankungen unterliegt. Als Beispiel: bei einem Patienten ist einer von $4 \mathrm{LK}$ positiv (Density entsprechend $25 \%$ ), bei einem anderen zeigten sich 4 von $40 \mathrm{LK}$ positiv (Density 10\%). Gemäß LK-Density hat der 1. Patient das höhere Risiko innerhalb von 10 Jahren an seinem Blasenkarzinom zu versterben. Hierbei gilt es jedoch $\mathrm{zu}$ bedenken, dass bei diesem Patienten sicherlich die Gesamtzahl der resezierten LK eine entscheidende Rolle spielt, da bei lediglich 4 resezierten LK davon auszugehen ist, dass etliche, vermutlich auch tumorpositive LK in situ verblieben sind. Auf der anderen Seite ist beim 2. Patienten die nodale Tumorlast deutlich höher.

Nicht nur LK-Anzahl respektive LKDensity sondern auch das Ausmaß der LK-Metastase werden als wichtige prognostische Faktoren diskutiert. Mills et al. [20] konnten zeigen, dass Patienten mit einem maximalen LK-Metastasendurchmesser von $0,5 \mathrm{~cm}$ im Median 64 Monate überlebten, hingegen solche mit einem LK-Metastasendurchmeser $>0,5 \mathrm{~cm}$ nur 16 Monate $(p=0,024)$. Eine der bis heute wohl wichtigsten prognostischen Parameter stellt das extrakapsuläre Wachstum dar, welches im Rahmen einer multivariaten Analyse den stärksten prädiktiven Wert ausmachte [8]. Hingegen konnten Kassouf et al. [14] diese Beobachtung im Rahmen ihrer Studie nicht bestätigen. Diese Studie basiert aber nur auf den den Autoren vorliegenden Pathologieberichten, eine neuerliche Aufarbeitung der Schnittpräparate erfolgte nicht. Es wird weiter angenommen, dass bei Nachweis von tumorpositiven LK mit extrakapsulärem Wachstum ein aggressiverer Tumortyp mit einer höheren Wahrscheinlichkeit und Geschwindigkeit der Metastasierung vorliegt. 
Hier steht eine Anzeige.

黛 Springer 


\section{Molekulares Lymphknotenstaging}

Bei der histopathologischen Aufarbeitung und Standarduntersuchung besteht letztlich immer die Gefahr, Mikrometastasen $\mathrm{zu}$ übersehen. Wir müssen uns bewusst sein, dass von einem einzelnen LK nur gerade 1 Querschnitt à $3 \mu \mathrm{m}$ (entsprechend ca. 1\%o) histologisch untersucht wird. Durch neue molekulare Marker und die entsprechenden Untersuchungstechniken verspricht man sich, dass die Sensitivität erhöht und damit das Staging verfeinert werden kann, was letztlich auch den Entscheid zu einer adjuvanten Therapie beeinflussen würde.

Kurahashi et al. [16] setzten eine Real-time-reverse-Transkriptase-PCR ein, um die Genexpression von Cytokeratin-19 (CK-19, ein im Epithel vorkommendes Protein) und Uroplakin II (Marker sowohl für normale als auch maligne urotheliale Zellen) an 40 zystektomierten und lymphadenektomierten Patienten zu untersuchen. Von 34 Patienten mit pN0Status waren 5 positiv für CK-19 und 10 für Uroplakin II. Bei diesen Patienten lag zudem ein prognostisch ungünstiger Faktor wie das Tumorstadium oder eine mikrovaskuläre Invasion vor. Die Bedeutung von Uroplakin II unterstreicht eine weitere Studie um Wu et al. [35], die zusätzlich noch CK-20 getestet, dieses aufgrund der Ergebnisse jedoch als weniger spezifisch eingestuft haben. Alle untersuchten Harnblasenpräparate $(n=19)$ wurden positiv auf Uroplakin II getestet und in 15 der 16 (93.8\%) pathologischen LK wurde ebenso Uroplakin-II-mRNA gefunden.

Stellvertretend für eine Vielzahl anderer molekularer Marker müssen die Ergebnisse zu Uroplakin II anhand weiterer Studien mit größeren Fallzahlen und längerem Follow-up untersucht werden. In der Zukunft sind auf molekularer Ebene eine genauere Diagnostik sowie basierend darauf eine bessere Risikostratifizierung und gleichzeitig Evaluierung bezüglich spezifischer Therapieansätze („targeted therapy“) denkbar und möglich.

\section{Minimal-invasive Verfahren und Lymphadenektomie}

Eine weitere Kontroverse bzgl. Ausdehnung der LAD tut sich durch den zunehmenden Trend zur minimal-invasiven Operationstechnik auch bei der radikalen Zystektomie auf. Werden durch laparoskopische respektive roboterassistierte Verfahren ein geringerer Blutverlust sowie eine Reduktion der perioperativen Morbidität zu erzielen versucht, so bleibt momentan noch die Frage, inwieweit die prognostisch relevante LAD an das offene operative Verfahren heranreicht. Die technischen Möglichkeiten zur LAD sind vorhanden, eine solche geht jedoch - wie auch in der offenen Chirurgie - mit einer deutlichen Verlängerung der Operationsdauer einher, was wiederum den minimal-invasiven Ansatz eines Verfahrens zumindest in Teilen in Frage stellt. Ghazi et al. [9] konnten mit einem supraumbilikal platzierten Trokar und Verwendung einer $30^{\circ}$-Optik durchschnittlich 25 (1932) LK/Patient resezieren.

Schumacher et al. [25] zeigten, dass die Zahl der resezierten LK respektive die Ausdehnung der LAD bei dem roboterassistierten Verfahren mit zunehmender Erfahrung bzw. Lernkurvenanstieg wächst. Eine retrospektive Studie des „International Robotic Cystectomy Consortiums“ bestätigt diese Daten an einem Kollektiv von insgesamt 513 Patienten, die im Zeitraum von 2003-2009 an 15 verschiedenen Zentren zystektomiert wurden [11]; 83\% dieser Patienten erhielten eine LAD mit einem Median von 17 (0-68) resezierten LK. Bei 43\% der Patienten wurden $>20$ LK reseziert. Auch hier zeigt sich aber eindrücklich, dass die Zahl der durchgeführten Eingriffe und die Erfahrung des Operateurs diesbezüglich von zentraler Bedeutung sind.

Letztlich bleibt die LAD ein zwingender Bestandteil der Zystektomie, der unabhängig von der Erfahrung des jeweiligen Operateurs und dem gewählten operativen Verfahren (offen, minimal-invasiv) akribisch im ausgedehnten Template durchgeführt werden muss.

\section{Ausblick}

Hinsichtlich der Evidenz einer LAD bei urologischen Tumoren liegt bis zum aktuellen Zeitpunkt lediglich eine prospektiv randomisierte Studie vor, die den Stellenwert der LAD bei der radikalen Nephrektomie analysiert [3]. Für die LAD im Rahmen der Zystektomie sind erfreulicherweise aktuell zwei prospektiv randomisierte Studien im Gange:

- Die sog. „LEA-Studie (Lymphaden-

ektomie eingeschränkt vs. ausge-

dehnt)“ der Arbeitsgemeinschaft urologische Onkologie (AUO) der Deutschen Krebsgesellschaft vergleicht das Outcome von Patienten mit radikaler Zystektomie und Standard-LAD (eingeschränkt: Fossa obturatoria, iliakal extern und intern) mit dem einer maximal ausgedehnten LAD (das gesamte lymphatische Gewebe vom Beckenboden bis zur A. mesenterica inferior inklusive der Fossa Marcille). Eingeschlossen wurden insgesamt $485 \mathrm{~Pa}$ tienten mit resektablem muskelinvasivem Urothelkarzinom der Harnblase ohne Nachweis pathologisch vergrößerter LK oberhalb der Aortenbifurkation und keiner vorgängigen neoadjuvanten Therapie. Es ist zu erwarten, dass das Outcome der Patienten mit einer maximal ausgedehnten LAD besser sein wird als das jener Patienten mit der Standard-LAD. Die Frage, wie weit die proximale Grenze der LAD (ausgedehnt vs. maximal ausgedehnt) gehen sollte, wird durch diese Studie jedoch nicht zu klären sein.

- Der „SWOG- (Soutwest Oncology Group-)Trial S1011“ startete vor kurzem und soll insgesamt $620 \mathrm{~Pa}$ tienten einschließen, wobei hier das Standardtemplate mit dem (eingeschränkt) ausgedehnten Template randomisiert verglichen wird. In diesem Trial wird als Standard-LAD die Resektion der LK perivesikal, obturatorisch, iliakal intern und extern bis zur Iliakalbifurkation definiert. Das ausgedehnte Template umfasst all diese eben erwähnten LK-Lokalisation und zudem präsakrale und iliakal kommune LK bis zur Aortenbifurkation sowie die LK der Fossa Marcil- 
le. Es ist eine 5-jährige Rekrutierungsphase gefolgt von einem 3-jährigen Follow-up geplant.

Die beiden Trials unterscheiden sich in bestimmten Punkten deutlich. So schließt der „SWOG-Trial“ u. a. neoadjuvant chemotherapierte Patienten ein und wird dies als eigenen Stratifikationsfaktor berücksichtigen. Die Einschlusskriterien differieren im Weiteren hinsichtlich des Tumorstadiums: Im Rahmen der „LEA-Studie“ werden Tumorstadien $\geq \mathrm{pT}$, im ,SWOGTrial“" Tumorstadien $\geq \mathrm{pT} 2$ eingeschlossen. Zuletzt zeigen sich auch bei der Definition des ausgedehnten Templates Unterschiede, da bei der „LEA-Studie“ LK bis proximal zur A. mesenterica inferior, hingegen beim "SWOG-Trial“ nur bis zur Aortenbifurkation hin reseziert werden. Die Ergebnisse beider Trials sind somit differenziert zu betrachten und nicht direkt miteinander vergleichbar.

\section{Fazit für die Praxis}

- Die LAD ist ein zwingender Bestandteil der radikalen Zystektomie hinsichtlich Staging, Therapie und Prognose. Dieser Grundsatz sollte unabhängig vom gewählten operativen Verfahren (offen vs. minimal-invasiv) gelten.

- Kontrovers diskutiert bleibt das optimale Template, wobei die vorliegenden retrospektiven Studien die limitierte oder gar unilaterale LAD als ungenügend einstufen.

- Die ausgedehnte LAD bis hin zum oberen Drittel der iliakal kommunen Gefäße inklusive Fossa Marcille scheint ein vergleichbares Outcome zu generieren wie das maximal ausgedehnte Template bis hin zur A. mesenterica inferior und sollte bis zum Vorliegen fundierter anderweitiger, prospektiv randomisierter Daten als Standard definiert werden.

- Die Zahl der resezierten LK ist kein hinreichendes Qualitätskriterium einer LAD, auch sind interinstitutionelle Vergleiche diesbezüglich wenig sinnvoll.

- Bezüglich der LK-Density bleibt zu diskutieren bzw. bleibt gleichzeitig fraglich, ob dieses Einzug in das pathologische Staging nehmen sollte.

- Im Weiteren gilt es, Studien bezüglich molekularer Marker hinsichtlich Staging, Prognose und allfälliger „,targeted therapy" zu forcieren.

\section{Korrespondenzadresse}

\section{T. Metzger}

Urologische Universitätsklinik, Inselspital,

Anna Seiler-Haus, CH-3010 Bern,

Schweiz

tobias.metzger@insel.ch

Interessenkonflikt. Der korrespondierende Autor gibt an, dass kein Interessenkonflikt besteht.

\section{Literatur}

1. Abol-Enein $\mathrm{H}, \mathrm{El}$-Baz M, Abd El-Hameed MA et al (2004) Lymph node involvement in patients with bladder cancer treated with radical cystectomy: a patho-anatomical study-a single center experience. J Urol 172:1818-1821

2. Baltaci S, Adsan O, Ugurlu O et al (2010) Reliability of frozen section examination of obturator lymph nodes and impact on lymph node dissection borders during radical cystectomy: results of a prospective multicentre study by the Turkish Society of Urooncology. BJU Int 107:547-553

3. Blom JH, Poppel H van, Marechal JM et al (2009) Radical nephrectomy with and without lymphnode dissection: final results of European Organization for Research and Treatment of Cancer (EORTC) randomized phase 3 trial 30881. Eur Urol 55:28-34

4. Brossner C, Pycha A, Toth A et al (2004) Does extended lymphadenectomy increase the morbidity of radical cystectomy? BJU Int 93:64-66

5. Dhar NB, Klein EA, Reuther AM et al (2008) Outcome after radical cystectomy with limited or extended pelvic lymph node dissection. J Urol 179:873878

6. Dorin RP, Daneshmand S, Eisenberg MS et al (2011) Lymph node dissection technique is more important than lymph node count in identifying nodal metastases in radical cystectomy patients: a comparative mapping study. Eur Urol 60:946-952

7. Fang AC, Ahmad AE, Whitson JM et al (2010) Effect of a minimum lymph node policy in radical cystectomy and pelvic lymphadenectomy on lymph node yields, lymph node positivity rates, lymph node density, and survivorship in patients with bladder cancer. Cancer 116:1901-1908

8. Fleischmann A, Thalmann GN, Markwalder R, Studer UE (2005) Extracapsular extension of pelvic lymph node metastases from urothelial carcinoma of the bladder is an independent prognostic factor. J Clin Oncol 23:2358-2365

9. Ghazi A, Zimmermann R, Al-Bodour A et al (2010) Optimizing the approach for lymph node dissection during laparoscopic radical cystectomy. Eur Urol 57:71-78

10. Ghoneim MA, Abol-Enein H (2004) Lymphadenectomy with cystectomy: is it necessary and what is its extent? Eur Urol 46:457-461
11. Hellenthal NJ, Hussain A, Andrews PE et al (2011) Lymphadenectomy at the time of robot-assisted radical cystectomy: results from the International Robotic Cystectomy Consortium. BJU Int 107:642646

12. Herr HW (2007) The concept of lymph node density-is it ready for clinical practice? J Urol 177:12731275

13. Kassouf W, Agarwal PK, Herr HW et al (2008) Lymph node density is superior to TNM nodal status in predicting disease-specific survival after radical cystectomy for bladder cancer: analysis of pooled data from MDACC and MSKCC. J Clin Oncol 26:121-126

14. Kassouf W, Leibovici D, Luongo T et al (2006) Relevance of extracapsular extension of pelvic lymph node metastasis in patients with bladder cancer treated in the contemporary era. Cancer 107:1491-1495

15. Kerr WS Jr, Colby FH (1951) Carcinoma of the bladder: a correlation of pathology with treatment and prognosis. J Urol 65:841-849

16. Kurahashi T, Hara I, Oka N et al (2005) Detection of micrometastases in pelvic lymph nodes in patients undergoing radical cystectomy for locally invasive bladder cancer by real-time reverse transcriptasePCR for cytokeratin 19 and uroplakin II. Clin Cancer Res 11:3773-3777

17. Leissner J, Hohenfellner R, Thuroff JW, Wolf HK (2000) Lymphadenectomy in patients with transitional cell carcinoma of the urinary bladder; significance for staging and prognosis. BJU Int 85:817823

18. Liedberg F, Chebil G, Davidsson T et al (2006) Intraoperative sentinel node detection improves nodal staging in invasive bladder cancer. J Urol 175:8488

19. May M, Herrmann E, Bolenz C et al (2011) Association between the number of dissected lymph nodes during pelvic lymphadenectomy and cancer-specific survival in patients with lymph nodenegative urothelial carcinoma of the bladder undergoing radical cystectomy. Ann Surg Oncol 18:2018-2025

20. Mills RD, Turner WH, Fleischmann A et al (2001) Pelvic lymph node metastases from bladder cancer: outcome in 83 patients after radical cystectomy and pelvic lymphadenectomy. J Urol 166:1923

21. Park J, Kim S, Jeong IG et al (2011) Does the greater number of lymph nodes removed during standard lymph node dissection predict better patient survival following radical cystectomy? World J Urol 29:443-449

22. Poulsen AL, Horn T, Steven K (1998) Radical cystectomy: extending the limits of pelvic lymph node dissection improves survival for patients with bladder cancer confined to the bladder wall. J Urol 160:2015-2019

23. Quek ML, Flanigan RC (2009) The role of lymph node density in bladder cancer prognostication. World J Urol 27:27-32

24. Roth B, Wissmeyer MP, Zehnder P et al (2009) A new multimodality technique accurately maps the primary lymphatic landing sites of the bladder. Eur Urol 57:205-211

25. Schumacher MC, Jonsson MN, Wiklund NP (2009) Does extended lymphadenectomy preclude laparoscopic or robot-assisted radical cystectomy in advanced bladder cancer? Curr Opin Urol 19:527532 
26. Seiler R, Gunten M von, Thalmann GN, Fleischmann A (2010) Pelvic lymph nodes: distribution and nodal tumour burden of urothelial bladder cancer. J Clin Pathol 63:504-507

27. Skinner DG (1982) Management of invasive bladder cancer: a meticulous pelvic node dissection can make a difference. J Urol 128:34-36

28. Stein JP, Cai J, Groshen S, Skinner DG (2003) Risk factors for patients with pelvic lymph node metastases following radical cystectomy with en bloc pelvic lymphadenectomy: concept of lymph node density. J Urol 170:35-41

29. Stein JP, Lieskovsky G, Cote R et al (2001) Radical cystectomy in the treatment of invasive bladder cancer: long-term results in 1,054 patients. J Clin Oncol 19:666-675

30. Stein JP, Penson DF, Cai J et al (2007) Radical cystectomy with extended lymphadenectomy: evaluating separate package versus en bloc submission for node positive bladder cancer. J Urol 177:876-881

31. Vazina A, Dugi D, Shariat SF et al (2004) Stage specific lymph node metastasis mapping in radical cystectomy specimens. J Urol 171:1830-1834

32. Weingartner $K$, Ramaswamy $A$, Bittinger $A$ et al (1996) Anatomical basis for pelvic lymphadenectomy in prostate cancer: results of an autopsy study and implications for the clinic. J Urol 156:19691971

33. Whitmore WF Jr, Marshall VF (1962) Radical total cystectomy for cancer of the bladder: 230 consecutive cases five years later. J Urol 87:853-868

34. Wiesner C, Salzer A, Thomas C et al (2009) Cancerspecific survival after radical cystectomy and standardized extended lymphadenectomy for nodepositive bladder cancer: prediction by lymph node positivity and density. BJU Int 104:331-335

35. Wu X, Kakehi Y, Zeng Y et al (2005) Uroplakin II as a promising marker for molecular diagnosis of nodal metastases from bladder cancer: comparison with cytokeratin 20. J Urol 174:2138-2142

36. Zehnder P, Studer UE, Skinner EC et al (2011) Super extended versus extended pelvic lymph node dissection in patients undergoing radical cystectomy for bladder cancer: a comparative study. J Urol 186:1261-1268

\section{Lieb K, Klemperer D, Ludwig W-D (Hrsg.) \\ Interessenkonflikte \\ in der Medizin}

Hintergründe und

Lösungsmöglichkeiten

Heidelberg: Springer 2011, 300 S., 13 Abb., (ISBN 978-3-642-19841-0), 61.00 EUR

Die Diskussion um Beziehungsgeflechte hat in der Öffentlichkeit häufig einen aktuellen Bezug. Im Berufsleben eines Arztes gibt es niemand ohne eine Berührung mit Interessenkonflikten.

Das Buch über "Interessenkonflikte in der Medizin" hat das Ziel, den Umgang mit diesen Problemen im Bereich zwischen Ärzten und Pharmaindustrie zu sichten. Aus dieser Motivation entstand ein Text, der die vielseitigen Facetten von Interessenkonflikten und deren Auswirkung in Forschung, Krankenversorgung, Aus-, Weiter- und Fortbildung behandelt. Darüber hinaus werden Rückwirkungen auf medizinische Fachzeitschriften und auf den Medizin-Journalismus dargestellt.

Interessenkonflikte werden definiert als Situationen, die ein Risiko dafür schaffen, dass professionelles Urteilsvermögen oder Handeln unangemessen beeinflusst wird.

Die häufig übliche Gleichsetzung von Interessenkonflikten mit Bestechlichkeit und Korruption kann nicht nur falsch, sondern auch schädlich sein für einen angemessenen Umgang mit Interessenkonflikten.

Das primäre Interesse der Ärzte ist es, für das Wohl der Patienten durch bestmögliche Diagnostik und Behandlung zu sorgen und das medizinische Wissen weiter zu entwickeln.

Sekundäre Interessen, z. B. materieller Art, können bis an die Grenze von Bestechlichkeit und Korruption gehen. Aber auch immaterielle Einflussnahmen - wie der Wunsch nach Anerkennung oder Förderung der Karriere usw. - können die ärztliche Tätigkeit beeinflussen.

In dem vorgelegten Buch wird die aktuelle Situation der internationalen Diskussion zusammengefassend dargestellt. Vertreter der verschiedenen Fachrichtungen, aber auch pharmazeutische Unternehmen kommen zu Wort.

Das Buch soll zu einer Versachlichung der Diskussion beitragen. Art und Umfang von ArztIndustrie-Kontakten werden angesprochen. Etwa 16.000 Pharmareferenten besuchen in Deutschland niedergelassene und klinisch tätige Ärzte. Neben wichtigen Fachinformationen werden Schreibwaren, Medikamentenproben, Essenseinladungen und Reisekosten zu Kongressen angeboten. Dabei ist eine Abgrenzung vertretbarer Interessen von Bestechung nicht immer einfach.

Offenlegung und Dokumentation können zur Reduktion und Vermeidung von Verpflichtungen führen und sollten Fehlverhalten im Sinne einer Korruption vermeiden helfen. Kooperationen zwischen Wissenschaftlern, Ärzten und pharmazeutischen Unternehmen bei Veröffentlichungen in Fachzeitschriften, bei Fortbildungsveranstaltungen etc. sind notwendig müssen aber kritisch betrachtet und bewertet werden.

Die Sichtweise des Vereins der forschenden Arzneimittelhersteller, die Probleme von Medizin-Journalisten und die Bedeutung der Unabhängigkeit der Zeitschriften werden in Einzelkapiteln sachkundig behandelt. Unabhängige Zeitschriften wie u.a. der Arzneimittelbrief. sind zu empfehlen und werden auch benannt. Entwicklungen der Arzneimittelausgaben sowie die verschiedenen Vergütungssysteme werden angesprochen.

Das vorlegende Buch durchleuchtet vorwiegend das weithin bekannte Beziehungsgeflecht zwischen Pharmaindustrie und Ärzten. Es kann zu einer Versachlichung des Themas beitragen, da Interessenkonflikte in der Medizin allgegenwärtig sind.

Die „Freiwillige Selbstkontrolle der Arztneimittelindustrie" hat bereits einige strikte Vorschriften formuliert. Grundregeln in Klinik und Praxis sind Transparenz insbesondere für Ausbildung, Weiterbildung und Fortbildung. Allerdings wurden konfliktreiche Themenkreise zwischen Verdienen (Pharmaindustrie) und Sparen(Krankenkassen) nicht angesprochen. Das interdisziplinär von namhaften Autoren bearbeitete Buch mit Hinweisen auf Möglichkeiten, Interessenkonflikten zu vermeiden, verdient einen großen Leserkreis.

Die stets aktuelle Dynamik dieses Problems lassen es wünschenswert erscheinen, dass alle Berufsgruppen im Gesundheitswesen aber auch Patienten und Selbsthilfegruppen sich mit dem Thema auseinandersetzen.

J. Sökeland (Berlin) 